\title{
The effect of size ratio on the sphere structure factor in colloidal sphere-plate mixtures
}

\author{
G. Cinacchi, ${ }^{1,2, a)}$ N. Doshi, ${ }^{1}$ S. W. Prescott, ${ }^{1}$ T. Cosgrove,${ }^{1}$ I. Grillo, ${ }^{3}$ P. Lindner, ${ }^{3}$ \\ J. S. Phipps, ${ }^{4}$ D. Gittins, ${ }^{5}$ and J. S. van Duijneveldt ${ }^{1, a)}$ \\ ${ }^{1}$ School of Chemistry, University of Bristol, Cantock's Close, Bristol BS8 1TS, United Kingdom \\ ${ }^{2}$ Departamento de Física Teórica de la Materia Condensada, Universidad Autónoma de Madrid, \\ Campus de Cantoblanco, 28049 Madrid, Spain \\ ${ }^{3}$ Institut Laue-Langevin, BP156, 6 rue Jules Horowitz, 38042 Grenoble Cedex 9, France \\ ${ }^{4}$ Imerys Minerals Ltd., Par Moor Centre, Par Moor Road, Par Cornwall PL24 2SQ, United Kingdom \\ ${ }^{5}$ Imerys Performance \& Filtration Minerals, 1732 N 1st Street, San Jose, California 95112, USA
}

(Received 13 August 2012; accepted 31 October 2012; published online 28 November 2012)

\begin{abstract}
Binary mixtures of colloidal particles of sufficiently different sizes or shapes tend to demix at high concentration. Already at low concentration, excluded volume interactions between the two species give rise to structuring effects. Here, a new theoretical description is proposed of the structure of colloidal sphere-plate mixtures, based on a density expansion of the work needed to insert a pair of spheres and a single sphere in a sea of them, in the presence or not of plates. The theory is first validated using computer simulations. The predictions are then compared to experimental observations using silica spheres and gibbsite platelets. Small-angle neutron scattering was used to determine the change of the structure factor of spheres on addition of platelets, under solvent contrast conditions where the platelets were invisible. Theory and experiment agreed very well for a platelet/sphere diameter ratio $D / d=2.2$ and reasonably well for $D / d=5$. The sphere structure factor increases at low scattering vector $Q$ in the presence of platelets; a weak reduction of the sphere structure factor was predicted at larger $Q$, and for the system with $D / d=2.2$ was indeed observed experimentally. At fixed particle volume fraction, an increase in diameter ratio leads to a large change in structure factor. Systems with a larger diameter ratio also phase separate at lower concentrations. (C) 2012 American Institute of Physics. [http://dx.doi.org/10.1063/1.4767722]
\end{abstract}

\section{INTRODUCTION}

Mixtures of colloidal particles of different sizes or shapes have been shown to undergo phase separation at sufficiently high concentration. Already at low concentrations, excluded volume interactions between the two species give rise to structuring effects, as for example found recently in sphere-plate mixtures. ${ }^{1}$ In that case, spheres, the majority component, tended to aggregate as the plate concentration was increased.

In previous work on mixtures of differently sized or shaped particles, it has been shown that if the size ratio of the different particles was sufficiently different from unity and both types of species were "hard," the smaller particle may act as a depletant, driving the larger particles together. ${ }^{2-10}$ This is known as the depletion attraction, explained by Asakura and Oosawa in the fifties, ${ }^{11,12}$ where the centers of mass of the small particles cannot access the depletion volumes, around each large particle. In essence, when one large particle approaches another, these excluded volumes overlap. As a result, the small species can now access a greater volume, and the resulting increase in entropy of the many small particles generates a depletion attraction between the large particles.

While being of interest from a basic statisticalmechanical viewpoint, the depletion effect also has important

\footnotetext{
a) Authors to whom correspondence should be addressed. Electronic addresses: giorgio.cinacchi@uam.es and J.S.van-Duijneveldt@bristol.ac.uk.
}

repercussions in all those applied fields that employ colloidal formulations. In the context of the present work in particular, this effect may have a bearing on paint formulations, where expensive pigment particles (titania) are often mixed with lower cost extenders such as kaolin clay particles.

The depletion attraction and subsequent phase behaviour is found to be very dependent on the size ratio of the two colloid diameters ${ }^{12}$ and the shapes of both types of colloids (e.g., see Refs. 10, 13 and 14).

With regards to the dependence on the size ratio, a classic example is a colloid-polymer mixture, in which adding small non-adsorbing polymers to a dispersion of colloidal particles induces attractive forces between the latter. ${ }^{4,15}$ Calculations ${ }^{16,17}$ and experiments ${ }^{18,19}$ proved that for polymer-to-colloid size ratios $q<0.3$, there is a coexistence region between a colloidal solid phase and a colloidal fluid phase and at $q>0.3$, there is a gas-liquid coexistence. A further phase is observed if $q$ is increased to 0.4 , giving a triple gas-liquid-solid coexistence. ${ }^{17,20}$ The maximum depth and range of the depletion attraction are controlled by the concentration and size of the depletant agent, respectively. ${ }^{12,21,22}$ Changing these factors will affect the phase behaviour of the colloidal mixtures and the interparticle structure.

The shape of colloidal particles also strongly affects the strength of the attraction. For example, the depletion attraction will be stronger between plate-like particles, in 
comparison to colloidal spheres of a similar diameter, induced by a depletant of the same size and concentration. ${ }^{9,10,13}$ If the plates are touching face-to-face, the free volume which becomes available to the depletants is much larger than for two spheres coming together. Similar particles, as investigated in this work but with different surface treatment, have been used in two different studies of alumina coated silica spheres and gibbsite $\left(\mathrm{AlOH}_{3}\right)$ platelets by Kleshchanok et al. ${ }^{9,}, 10$ in which the sphere particles were found to deplete the platelet particles, at high volume fractions. Kleshchanok et al. showed that by doubling the platelet diameter and keeping the sphere diameter constant, that is doubling the size ratio, the effective attraction increases. The phase behaviour changed from co-existing isotropic-columnar phases at the lower size ratio ${ }^{9}$ to co-existing isotropic-glass phase at the higher size ratio. ${ }^{10}$ The glass phase was caused by the deep depletion potential but did contain domains of liquid crystals that became more sporadic when the concentration of the spheres was increased.

In this work, we investigate colloidal mixtures of spheres and plates at low volume fractions, so that the suspensions remain homogeneous; the study focuses on how the structure of the spheres depends on the sphere/plate size ratio upon addition of plates. Experimentally, this is probed using smallangle neutron scattering (SANS), using a solvent composition that matches the scattering length density (SLD) of the platelets. A preliminary version of this work showed that indeed addition of platelets induces an increase of structure factor (clustering) of the spheres, ${ }^{1}$ in analogy with what happens when large polymers are added to small colloids ("protein limit"). ${ }^{23-26}$ An elementary theoretical model, valid in the very low density and low plate/sphere size ratio limits, offered justification for this behaviour but, to achieve a satisfactory fit to the data, unrealistically small values for the platelet diameter had to be used. Here, an improved version of the theory is presented and validated using computer simulations. The theory is valid at higher concentrations than the earlier treatment. New experimental data are presented for the same type of platelets, with thickness-to-diameter ratio $L / D=0.08$, mixed with three different sizes of spheres, designed to afford a stringent test of the theory. Agreement is good for mixtures with platelet/sphere diameter ratio $D / d=2.2$, and satisfactory for $D / d=5$, whereas the smallest spheres scattered too weakly to allow detailed analysis. The data demonstrate the broad trend that the effect on the structure of the spheres, at fixed sphere and platelet volume fraction, increases with $D / d$.

\section{THEORY}

Let us consider a suspension of hard spheres of diameter $d$ and number density $\rho_{\circ}$. The sphere-sphere radial distribution function, characterising the structure of this suspension, is $g_{\circ \circ}^{\circ}(r)$, with $r$ the distance separating the two spheres (e.g., Ref. 27). The superscript $\circ$ indicates that the radial distribution function refers to a pure suspension of spheres. Upon the addition of other particles, e.g., platelets, to the sphere suspension, $g_{\circ \circ}^{\circ}(r)$ will change. Let us label the modified spheresphere radial distribution function $g_{\circ \circ}^{P}(r)$. The superscript $P$ is chosen because the focus of this work is on the addition of plate-like particles; nonetheless, the following discussion is not specific to the shape of the added particles. We seek a theory to relate $g_{\circ \circ}^{P}(r)$ to $g_{\circ \circ}^{\circ}(r)$, with $\rho_{P}$ the number density of the added particles.

For hard spheres, the radial distribution function coincides, for $r \geq d$, with the cavity-cavity distribution function. ${ }^{28}$ This one, labeled either $y_{\circ \circ}^{\circ}(r)$ or $y_{\circ \circ}^{P}(r)$, respectively, is interpreted as the probability density to find a cavity of diameter $d$ at a distance $r$ from another cavity of the same size. Any cavity-cavity distribution function, $y_{\circ \circ}^{s}(r)$, with $s=\circ$ or $P$, is linked to the difference of the work needed to create two cavities at distance $r, w_{\circ \circ}^{s}(r)$, with twice the work needed to create a single cavity

$$
y_{\circ \circ}^{s}(r)=\exp \left[-\beta\left(w_{\circ \circ}^{s}(r)-2 w_{\circ}^{s}\right)\right]
$$

with $\beta=1 /\left(k_{B} T\right), k_{B}$ and $T$ being the Boltzmann constant and temperature, respectively.

Both types of work are needed in the presence of the added platelets and are related to the corresponding work needed without them

$$
\begin{gathered}
w_{\circ \circ}^{P}(r)=w_{\circ \circ}^{\circ}(r)+w_{\circ \circ}^{P \circ}(r), \\
w_{\circ}^{P}=w_{\circ}^{\circ}+w_{\circ}^{P \circ}
\end{gathered}
$$

with $w_{\circ \circ}^{P \circ}(r)$ and $w_{\circ}^{P \circ}$ the additional work needed to find, respectively, a pair of cavities and a single cavity when platelets are present.

The decomposition of Eq. (2) leads to the following relationship between $y_{\circ \circ}^{P}(r)$ and $y_{\circ \circ}^{\circ}(r)$ :

$$
y_{\circ \circ}^{P}(r)=y_{\circ \circ}^{\circ}(r) \exp \left[-\beta\left(w_{\circ \circ}^{P \circ}(r)-2 w_{\circ}^{P \circ}\right)\right] .
$$

If $\rho_{P}$ is sufficiently small, both the additional work contributions are small as well and this implies that:

(i) just the leading term of the exponential of Eq. (3) can be retained:

$$
y_{\circ \circ}^{P}(r) \simeq y_{\circ \circ}^{\circ}(r)\left[1-\beta\left(w_{\circ \circ}^{P \circ}(r)-2 w_{\circ}^{P \circ}\right)\right] ;
$$

(ii) an expansion of the additional work contributions in a power series of densities can be truncated at the first two terms.

In the density expansion of the additional work contributions, the first term is proportional to $\rho_{P}$ while the successive term is proportional to $\rho_{P} \rho_{\circ}$. The explicit expression for the additional work required to insert a pair of spheres in the presence of plates becomes

$$
\beta w_{\circ \circ}^{P \circ}(r)=\rho_{P} \Omega_{\circ}^{(1)}(r)+\rho_{P} \rho_{\circ} \Omega_{\circ}^{(2)}(r),
$$

where

$$
\Omega_{{ }_{\circ}}^{(1)}(r)=-\int d \mathbf{R} d \hat{\mathbf{u}} \mathrm{f}_{{ }_{\circ}} P(\mathbf{R}, \hat{\mathbf{u}})
$$

with $f_{\circ} P$ the Mayer function of a dumbbell formed by two hard spheres separated by a distance $r$ and a hard platelet, $\mathbf{R}$ the distance vector between their centers, and $\hat{\mathbf{u}}$ the unit vector describing the orientation of the platelet with respect to the 
dumbbell's main axis. Furthermore,

$$
\begin{aligned}
\Omega_{\circ}^{(2)}(r)= & -\int d \mathbf{R} d \hat{\mathbf{u}} d \mathbf{R}^{\prime} \mathrm{f}_{\circ} P(\mathbf{R}, \hat{\mathbf{u}}) \\
& \times \mathbf{f}_{\circ}{ }_{\circ}\left(\mathbf{R}, \mathbf{R}^{\prime}\right) \mathbf{f}_{\circ P}\left(\mathbf{R}, \hat{\mathbf{u}}, \mathbf{R}^{\prime}\right),
\end{aligned}
$$

with $f_{\circ}\left(\mathbf{R}, \mathbf{R}^{\prime}\right)$ and $\mathbf{f}_{\circ}\left(\mathbf{R}, \hat{\mathbf{u}}, \mathbf{R}^{\prime}\right)$, respectively, the Mayer function of the dumbbell and a sphere and that one of a platelet and that sphere.

The additional work to insert a single sphere in the presence of plates becomes

$$
\beta w_{\circ}^{P \circ}=\rho_{P} \omega_{\circ P}^{(1)}+\rho_{P} \rho_{\circ} \omega_{\circ P}^{(2)}
$$

with

$$
\omega_{\circ P}^{(1)}=-\int d \mathbf{R} \mathbf{f}_{\circ P}(\mathbf{R}),
$$

where $f_{\circ P}(\mathbf{R})$ is the Mayer function between a sphere and a platelet located at a distance $\mathbf{R}$ from its center. Finally,

$$
\omega_{\circ P}^{(2)}=-\int d \mathbf{R} d \mathbf{R}^{\prime} \mathfrak{f}_{\circ P}(\mathbf{R}) \mathfrak{f}_{\circ P}\left(\mathbf{R}^{\prime}\right) \mathfrak{f}_{\circ \circ}\left(\left|\mathbf{R}-\mathbf{R}^{\prime}\right|\right)
$$

with $\mathrm{f}_{\circ \circ}\left(\left|\mathbf{R}-\mathbf{R}^{\prime}\right|\right)$ the Mayer function between two spheres whose centers are separated by a distance $\left|\mathbf{R}-\mathbf{R}^{\prime}\right|$.

Put together, Eqs. (4)-(10) form the improved theory. It is convenient to distinguish between it and the lowest order theory, already presented in the previous article and here recovered by retaining in Eqs. (5) and (8) only the first term with the (1) superscript. Let us define the difference $\beta\left(w_{\circ \circ}^{P \circ}(r)\right.$ $\left.-2 w_{\circ}^{P \circ}\right)$ in this case as $\Delta v_{I}(r)$. By analogy, the same difference as obtained by the second-order theory, where terms with superscript (1) and (2) are both included, is defined as $\Delta v_{I I}(r)$. Section III describes the computer simulations performed to validate this theory.

\section{COMPUTER SIMULATIONS: VALIDATION OF THEORY}

Standard canonical ensemble (NVT) Monte Carlo (MC) computer simulations were run. The systems consisted of either $\mathrm{N}=500$ or 1000 hard spheres of diameter $d$. Four values of density, measured in reduced units, $\rho_{\circ}^{*}=\rho_{\circ} d^{3}$, were considered: $0.025,0.0625,0.125$, and 0.25 , all pertaining to the very dilute/dilute range of densities. Simple cubic lattice structures were used as starting configurations, which rapidly melted. To ensure that no influence of the initial conditions was left, equilibration runs lasted $500 \times 10^{3}$ cycles and were followed by a production run of the same length, each cycle consisting of $\mathrm{N}$ attempts to translate a randomly selected hard sphere by a random small amount along the three axes of the laboratory frame of reference, accepting the trial move if no overlap was detected. During each production run, the radial distribution function, $g_{\circ \circ}^{\circ}(r)$, was calculated by sampling and averaging histograms with a bin width of $0.01 d$.

Subsequently, to each of the four simple cubic lattice structures, 10 hard and infinitely thin platelets were manually added, taking due care to avoid overlap with any hard sphere. Two series of such starting configurations were considered, one with platelets having an area $\frac{\pi}{4} D^{2}=5 d^{2}$ and the

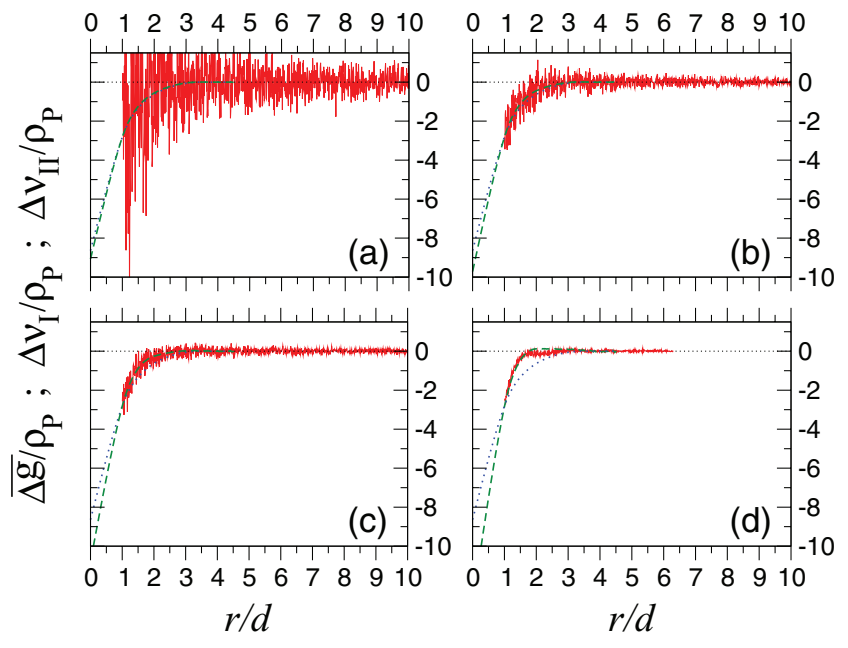

FIG. 1. The functions $\frac{\Delta \bar{g}(r)}{\rho_{P}}$ (red solid), $\frac{\Delta v_{I}(r)}{\rho_{P}}$ (blue dotted), $\frac{\Delta v_{I I}(r)}{\rho_{P}}$ (green dashed) obtained at various sphere densities $\rho^{*}=0.025$ (a), $\rho_{P} 0.0625$ (b), 0.125 (c), and 0.25 (d) for the case with platelets having a diameter $D=2 \sqrt{5 / \pi} d$.

other with platelets having an area $\frac{\pi}{4} D^{2}=20 d^{2}$. These starting configurations were used to run additional MC-NVT simulations. These too were organized in cycles, this time each of them consisting of $\mathrm{N}+10$ attempts to randomly pick up a particle and translate it by a random small amount if it was a sphere, or to translate and rotate it by random small amounts if it was a platelet. In the latter case, rotations were performed by rotating the randomly selected platelet's main axis around one of the three axes of the laboratory frame of reference, this was also selected at random. Trial moves were accepted if no sphere-sphere, sphere-platelet, and platelet-platelet overlaps were detected. Again, equilibration and production runs lasted $500 \times 10^{3}$ cycles. The sphere-sphere radial distribution function, $g_{\circ \circ}^{P}(r)$ was calculated by sampling and averaging histograms of the same bin size as before. The function

$$
\frac{\Delta \bar{g}(r)}{\rho_{P}}=\frac{1}{\rho_{P}}\left[1-\frac{g_{\circ \circ}^{P}(r)}{g_{\circ \circ}^{\circ}(r)}\right]
$$

was then obtained, which is to be compared to the expression coming from theory

$$
\frac{\Delta v_{I}(r)}{\rho_{P}} \text { and } \frac{\Delta v_{I I}(r)}{\rho_{P}} .
$$

Figures 1 and 2 provide this comparison, where distance is expressed in terms of the sphere diameter $d$. While the performance of the lower order theory is good at the lowest density investigated, it progressively deteriorates, as expected, as the sphere density increases. The figures demonstrate the new expressions agree very well with the simulations for sphere volume fractions up to $\phi_{\circ}=\frac{\pi}{6} \rho^{*} \simeq 0.07$ and deviate from them for $\phi_{\circ} \simeq 0.13$ slightly, in the case of smaller diameter platelets, and a bit more visibly, in the case of larger diameter platelets, being thus able to overall cover the range of concentrations studied experimentally. 


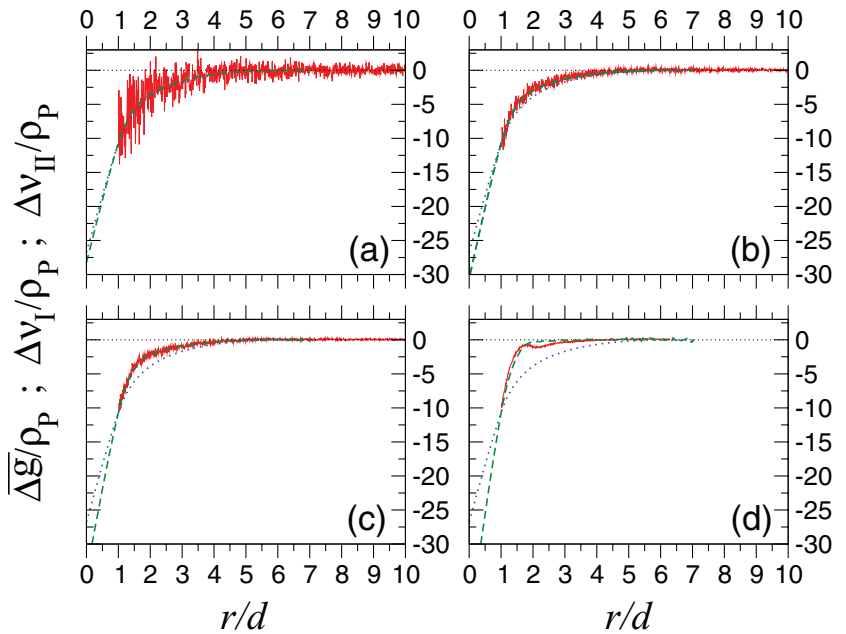

FIG. 2. The functions $\frac{\Delta \bar{g}(r)}{\rho_{P}}$ (red solid), $\frac{\Delta v_{I}(r)}{\rho_{P}}$ (blue dotted), $\frac{\Delta v_{I I}(r)}{\rho_{P}}$ (green dashed) obtained at various sphere densities $\rho^{*}=0.025$ (a), $\rho_{P} 0.0625$ (b), 0.125 (c), and 0.25 (d) for the case with platelets having an area $D=4 \sqrt{5 / \pi} d$.

\section{COMPARISON OF THEORY WITH EXPERIMENTS}

The theory developed and validated so far applies to monodisperse hard spheres to which hard platelets have been added. Experimental systems are not monodisperse, nor do their constituent particles have a regular shape and interact through perfectly hard-body interactions. Nonetheless, the relatively low degree of polydispersity of the experimental systems under consideration, together with the confidence that the particles are, within a good approximation, behaving as hard objects, make a direct comparison of the present theory to the experimental data reasonable. The theoretical approach could in principle be extended to polydisperse systems but this has not been pursued yet.

The relationship between the radial distribution function, $g(r)$, obtained in theory, and the structure factor, $S(Q)$, obtained in experiments, is well known. ${ }^{27}$ For a fluid system of spherical particles, it reads

$$
S(Q)=1+\frac{4 \pi \rho_{\circ}}{Q} \int_{0}^{\infty} d r r[g(r)-1] \sin (Q r) .
$$

The validated second-order theory can be used to predict the changes occurred to sphere structure factor upon the addition of platelets. From Eq. (13) and given the relationship between $g_{\circ \circ}^{P}(r)$ and $g_{\circ \circ}^{\circ}(r)$ provided by Eqs. (4)-(10), one arrives at

$$
\begin{aligned}
\Delta S_{\circ \circ}^{P \circ}(Q)= & S_{\circ \circ}^{P}(Q)-S_{\circ \circ}^{\circ}(Q) \\
& =\frac{4 \pi \rho_{\circ}}{Q} \int_{D+d}^{d} d r r g_{\circ \circ}^{\circ}(r) \Delta v_{I I}(r) \sin (Q r)
\end{aligned}
$$

with $S_{\circ \circ}^{P}$ and $S_{\circ \circ}^{\circ}$ the sphere structure factor with and without platelets, respectively. As an aside, the value of $\Delta S_{\circ \circ}^{P \circ}$ at $Q=0$ can also be obtained via a different route, using Kirkwood-Buff theory of mixtures, ${ }^{29,30}$ and this is shown in the Appendix.

For the purpose of comparison with experimental data, it is useful to introduce the function $Z(Q)$, defined as

$$
Z(Q)=\frac{\Delta S_{\circ \circ}^{P \circ}(Q)}{\phi_{P} S_{\circ \circ}^{\circ}(Q)},
$$

the usage of which requires knowing $S_{\circ \circ}^{\circ}(Q)$, obtainable from Eq. (13) once an expression for $g_{\circ \circ}^{\circ}(r)$ is known. This was provided by the exact solution of the Percus-Yevick integral equation theory for hard spheres, accurate over a wide range of sphere density. ${ }^{27}$

In the experiments, both sphere and platelet samples are polydisperse. In order to take this, at least partially, into account, for both series of experiments, three theoretical curves were calculated and averaged, as detailed below.

\section{EXPERIMENTAL}

In this work, the mineral particles [Ludox CL (Sigma Aldrich), Klebosol 30CAL25 and Klebosol 30CAL50 (AZ Electronics), gibbsite] were dialysed against $5 \mathrm{mM} \mathrm{NaCl}$ solution and stabilised with a commercial stabilizer [Solsperse 41000 (Lubrizol)]. The particle dimensions (bare and effective) are given in Table I (see Ref. 1 for details). The sphere/platelet size ratio was varied by comparing mixtures of one batch of gibbsite plates with spheres of different diameters, which for convenience will be referred to as particles A, $\mathrm{B}$, and $\mathrm{C}$. The effective dimensions, which take into account the Debye length $\left(\kappa^{-1}\right)$ and adsorbed polymer layer on the bare particles, are important as the effective diameter of the spheres and the effective volume fraction of both particles are used in calculating and predicting $Z(Q)$. The effective volume fractions $\left(\phi_{\mathrm{S}}^{\text {eff }}\right)$ of all three sphere types was kept constant at $\sim 0.1$, while the effective volume fractions of the plates $\left(\phi_{\mathrm{P}}^{\mathrm{eff}}\right)$ were varied (Ref. 1 describes how effective volume fractions

\begin{tabular}{|c|c|c|c|c|c|c|}
\hline \multirow[b]{2}{*}{ Particle } & \multirow[b]{2}{*}{ Code } & \multicolumn{2}{|c|}{ TEM/AFM } & \multicolumn{3}{|c|}{ Scattering data } \\
\hline & & Dimension & $\begin{array}{c}\text { Mean } \\
\mathrm{nm}\end{array}$ & $\begin{array}{c}\text { Polydispersity } \\
\%\end{array}$ & $\begin{array}{c}\text { Bare } \\
\mathrm{nm}\end{array}$ & $\begin{array}{c}\text { Effective } \\
\mathrm{nm}\end{array}$ \\
\hline Ludox CL & A & $\langle d\rangle$ & 18 & 17 & 17 & 25 \\
\hline Klebosol 30CAL25 & B & $\langle d\rangle$ & 30 & 15 & 30 & 40 \\
\hline Klebosol 30CAL50 & $\mathrm{C}$ & $\langle d\rangle$ & 74 & 21 & 74 & 90 \\
\hline Gibbsite-3 & & $\langle D\rangle$ & 186 & 29 & 180 & 188 \\
\hline Gibbsite-3 & & $\langle L\rangle$ & 5 & 20 & 6 & 15 \\
\hline
\end{tabular}
were estimated).

TABLE I. Mean dimensions of the spheres and plates, including polydispersity from TEM/AFM data and average bare and effective dimensions of spheres and plates from scattering data. 
All experiments were conducted on the D11 diffractometer at the high flux reactor of the Institut Laue-Langevin (ILL) in Grenoble, France. A neutron wavelength of $\lambda=10 \AA$ and three sample-detector positions of 2,8 , and $39 \mathrm{~m}$ were used to access a wave vector range of $Q=(4 \pi / \lambda) \sin (\theta / 2)$ $=0.001 \AA^{-1}$ to $0.25 \AA^{-1}$. All spectra were normalized and corrected by subtracting the scattering of the pure solvent, the empty cell as well as the small residual scattering of the gibbsite platelets, where appropriate. All samples were studied at $20{ }^{\circ} \mathrm{C}$.

By using $\mathrm{H}_{2} \mathrm{O}$ and $\mathrm{D}_{2} \mathrm{O}$ mixtures, the scattering length density of the solvent could be matched to that of the gibbsite plates, rendering the colloidal plates invisible. This required a composition of $41.5 \%$ by volume $\mathrm{D}_{2} \mathrm{O}$. This allowed changes in the effective structure factor of the silica spheres, $S_{\circ \circ}^{\circ}(Q)$, on addition of the plates to be analysed. Alumina coated silica spheres were kept at a constant concentration as the volume fraction of gibbsite plates was varied. The experimental volume fractions, $\phi_{\mathrm{S}}$ and $\phi_{\mathrm{P}}$, of the bare sphere and plates, respectively, can be calculated from the mass fractions using the particle densities; $\rho_{\text {silica }}=2.30 \mathrm{~g} \mathrm{~cm}^{-3}$ and $\rho_{\text {gibbsite }}=2.42 \mathrm{~g} \mathrm{~cm}^{-3}$.

The concentration of the particles were kept below the isotropic-nematic (I-N) phase transition of the plate particles. The I-N phase diagrams for all 3 sphere sizes as a function of the silica and gibbsite concentration are shown elsewhere. ${ }^{31}$ In order to compare the results at different size ratios, $\phi_{\mathrm{S}}^{\text {eff }}$ for all three sphere sizes was kept constant at $\sim 10 \% \mathrm{v} / \mathrm{v}$. However, as the effective diameter of particles $\mathrm{A}$ and $\mathrm{C}$ could not be measured before the samples were prepared, estimates were made for the effective diameter. The actual effective diameter when determined by SANS results meant the actual $\phi_{\mathrm{S}}^{\text {eff }}$ for particles $\mathrm{A}$ and $\mathrm{C}$ were $8 \% \mathrm{v} / \mathrm{v}$ and $12 \% \mathrm{v} / \mathrm{v}$, respectively.

\section{RESULTS AND DISCUSSION}

The SANS data are collected in Fig. 3. Note that, while the effective volume fractions were all close to 0.1 , this implied different "bare" volume fractions of the differently sized spheres. Although the SANS technique has been proven to reveal changes in the structure of the colloidal spheres, there are limitations to the technique regarding the sphere size.

Unfortunately, the smallest A spheres were beyond the sensitivity of SANS (see Figure 3(a)). There were two contributing factors: the low volume fractions required for a homogeneous suspension, and the low scattering intensity associated with the small sphere size (proportional to the cube of the particle size, at a fixed particle volume fraction). The phase diagrams for the sphere-plate mixtures (Ref. 31) reveal the reduced volume fractions required for an isotropic dispersion when the sphere size is reduced. Rather low volume fractions of particles A and gibbsite were employed in this experiment to avoid the formation of nematic droplets and also of any aggregates. Even at low concentrations, the mixtures with particles A were observed by optical microscopy to contain small aggregates. The sphere-plate mixture appeared extremely unstable at the high size ratio; whether this is due only to the increase in the effective attraction cannot be confirmed. One would expect the formation of liquid crystal structures as
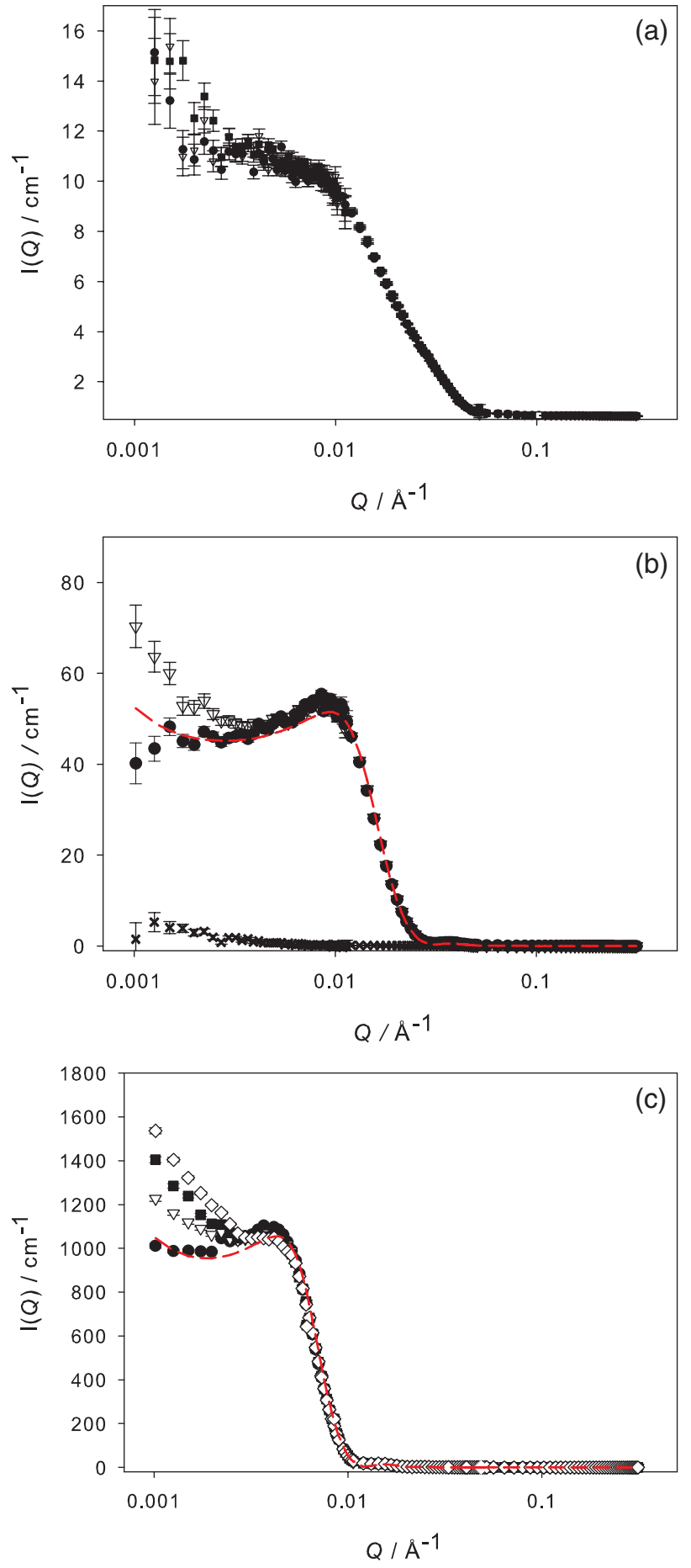

FIG. 3. SANS scattering curves for sphere-gibbsite mixtures in $41.5 \% \mathrm{v} / \mathrm{v}$ $\mathrm{D}_{2} \mathrm{O}$. (a) Particles A at $2.5 \% \mathrm{v} / \mathrm{v}$ only $(\bullet)$ and with $0.05 \% \mathrm{v} / \mathrm{v}(\nabla)$ and $0.1 \% \mathrm{v} / \mathrm{v}$ (ם) gibbsite plates; (b) particles B at $4.2 \% \mathrm{v} / \mathrm{v}$ only $(\bullet)$, and with $0.25 \% \mathrm{v} / \mathrm{v}$ gibbsite plates $(\nabla)$. For comparison, the scattering of $0.75 \% \mathrm{v} / \mathrm{v}(\mathbf{x})$ gibbsite plates is shown; (c) particles C at $6.7 \% \mathrm{v} / \mathrm{v}(\bullet)$ and with $0.25 \%(\nabla), 0.50 \%$ (घ) and $0.75 \% \mathrm{v} / \mathrm{v}(\diamond)$ gibbsite plates. Dashed lines are fits to the sphere-only data.

the mixture phase separates; however, the aggregates did not appear to be birefringent tactoids, but this may be due to their small size. Any structure that can be observed by microscopy would have an adverse impact on the scattering profiles at the vital length scales due to the excessive scattering of aggregates at low $Q$. The scattering data obtained for spheres A 

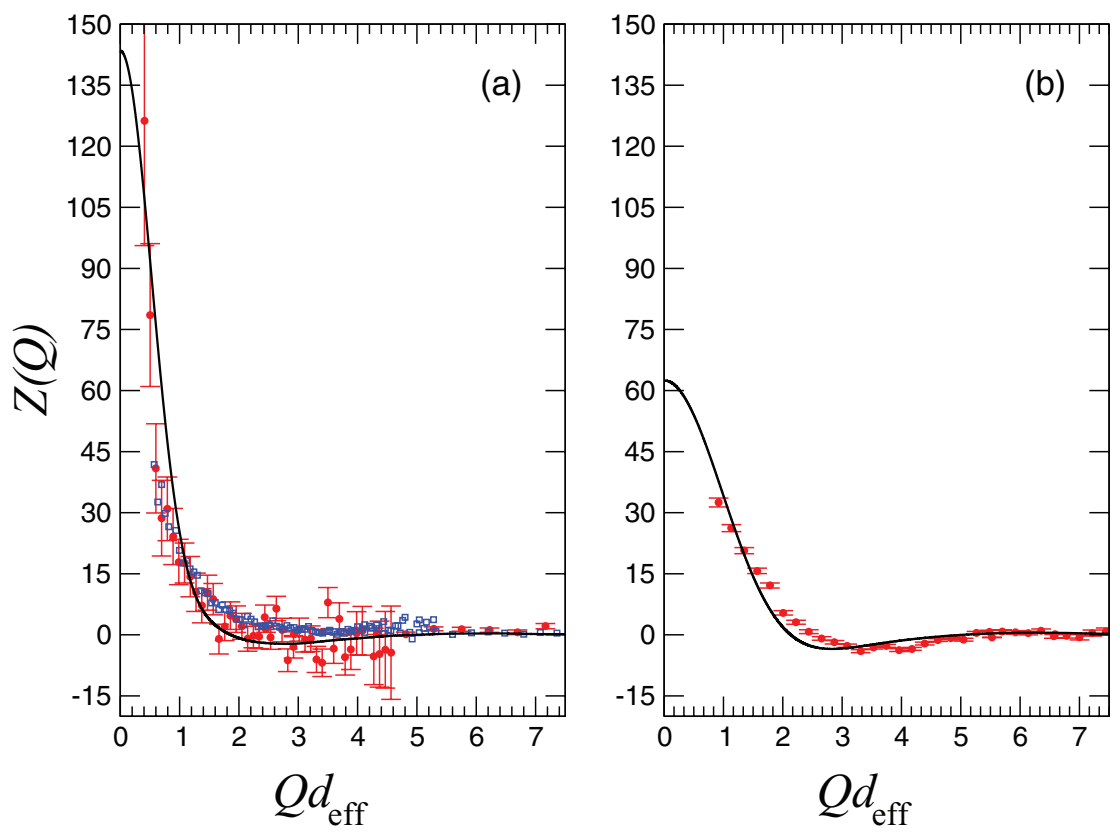

FIG. 4. Comparison between theory and experiments: (a) theoretical curve (black line) and experimental data (red circles (current) and blue squares (previous)) for particles B; (b) theoretical curve (black line) and experimental data (red circles) for particles C.

with and without the plates, shown in Figure 3(a), were noisy and have very low scattering intensity and no meaningful information on $Z(Q)$ could be extracted from these.

The situation is more encouraging for Sample B. Experiments using these particles were reported before in Ref. 1 and were repeated here, however with a different batch of gibbsite plates. The result are shown in Figure 3(b) and, as expected when $0.25 \% \mathrm{v} / \mathrm{v}$ of the gibbsite plates were added, an increased intensity was found at low $Q$. The sphere size from the SANS scattering profile of particles B is consistent with that found previously. Also, the scattering of the aqueous gibbsite dispersion in $41.5 \% \mathrm{v} / \mathrm{v}_{2} \mathrm{O}$ confirmed the near-perfect SLD matching of the plates under these solvent conditions.

To analyse the reproducibility of the change in the structure factor upon the addition of plates, $Z(Q)$ (Eq. (15)) was determined (Figure 4(a)). The agreement between the original and the repeated experiment is excellent. There are large error bars for the repeated scattering measurements, but this is expected as only one concentration of plates was measured so the data could not be averaged, and the neutron wavelength was longer, reducing the scattering intensity. However, data for both experiments overlay each other exactly giving significant confidence in the results and the reproducibility of the SANS technique.

The results obtained for particles $\mathrm{C}$ are in stark contrast to those for particles A. The increase in sphere size and the larger volume fraction available to formulate a homogeneous dispersion made the SANS experiment straightforward. The scattering profiles obtained (Figure 3(c)) were of a very high quality. The increase in scattering intensity of the spheres on the introduction of the plates is easily observed. This upswing in intensity is again found at low $Q$ as expected, due to an increase in the sphere structuring. Furthermore, the increase in the structure factor $S_{\circ \circ}^{\circ}(Q)$ is proportional to the plate con- centration as predicted by the theory. This is substantiated by the master curve of $Z(Q)$ obtained (Figure 4(b)), in which the increase in intensity is divided by the scattering intensity of the pure sphere suspension and by the effective plate concentration.

The theory presented in Secs. II-IV predicts that there will be a significant increase in structuring of the spheres as the size ratio $(D / d)$ increases. Therefore, it is expected that the larger spheres will have a lower $Z(Q)$ at low $Q$. In Figure 4 , the $Z(Q)$ for sphere-plate mixtures utilising particles $\mathrm{C}$, which have a size ratio $(D / d)$ of about 2 , are shown side by side with the previous results (Ref. 1) of sphere-plate mixtures containing particles B with a size ratio of about 5 . The effective diameters of the particles are used to calculate the size ratio. It is obvious that by reducing the size ratio, there is a significant reduction in the structuring of the spheres. The minimum in $Z(Q)$, which precedes the low $Q$ increase, moves to lower values of $Q$ as $d$ increases, and this is suggestive of structuring on a longer length scale. This implies an increased range in the effective attraction between the spheres with increasing sphere size. Parallels can be drawn to the AO theory, that says the range of the attraction is dependent on the size of the depletant. ${ }^{12,21,22}$ If we assume that the spheres act as the depletant, the range of attraction between the plates will increase with the sphere size. If the range in attraction between the plates particles increases consequentially, one may expect the range of the attraction between the sphere particles to also increase. Both changes in structuring and the range of the attraction due to the size ratio are expected and are in line with the new theoretical prediction, as shown by the close agreement between theoretical curves and experimental data in Figure 4.

In order to compare with the experiments, theoretical curves were averaged for three diameter ratios to account for particle polydispersities. Setting the sphere diameter as 
the unit of length, the values of plate diameter, $D^{*}=D / d$, and thickness, $L^{*}=L / d$, used were: $D^{*}=4.0 ; 5.0 ; 6.0$ and $L^{*}=0.2$ for comparison with the experimental data obtained using particles $\mathrm{B}$; and $D^{*}=1.5 ; 2.0 ; 2.5$ and $L^{*}=0.1$ for comparison with the experimental data obtained using particles $\mathrm{C}$. In the former case, the value of the sphere volume fraction used was 0.1 while a value of 0.12 was used in the latter case.

The density fluctuations observed in Figure 3 are caused by an (effective) attraction between the spheres. The length scales at which the increase in structuring occurs agree well with the theory. The length scales and the range of the interactions between the particles will be dependent on both particle sizes. In Figure 4(b), an unusual feature is observed for $Q$ between $2 \times 10^{-3}$ and $4 \times 10^{-3} \AA^{-1}$ (corresponding to $Q d_{\mathrm{eff}}=2$ - 4); the large $90 \mathrm{~nm}$ spheres produce a negative $Z(Q)$. This trait in $Z(Q)$ for the large spheres is in close agreement with the theoretical predictions. Experimentally, this occurs at a wavevector $Q$ corresponding to a length scale $\approx 2 d_{\text {eff. }}$ For the smaller spheres (Fig. 4(a)), the theory predicts there should still be a minimum, albeit a noticeably smaller one than for the large spheres; experimentally, no pronounced minimum is seen. This may be a result of the larger value for $L / d$ in this case. In fact, theory predicts that the depth of the minimum is mainly determined by this ratio, increasing as $L / d$ decreases. Theory also explains the negative minimum as a consequence of the damped oscillatory character around the $Q$ axis of the function $\Delta S_{\circ \circ}^{P \circ}(Q)$, which constitutes the numerator of the fraction leading to the function $Z(Q)$, the corresponding denominator being always positive. Particle polydispersity or, possibly, the softness of the particle interaction potentials may also contribute to the actual overall form of $Z(Q)$.

\section{CONCLUSION}

In this work, a new theoretical description of the structure of colloidal sphere-plate mixtures has been proposed and validated using computer simulations. By rendering the platelets effectively invisible, SANS allowed a detailed experimental test of this theory by determining the change of the structure factor of spheres on addition of platelets. New experimental data reproduced earlier data very well indeed. ${ }^{1}$ Theory and experiment agreed very well for $D / d=2.2$ and reasonably well for $D / d=5$. The smallest spheres induced phase separation or aggregation at low concentrations and also gave too little scattering intensity to allow a detailed comparison with theory. The sphere structure factor increases at low $Q$ in the presence of platelets; a weak reduction of $S(Q)$ was predicted at larger $Q$ and, for the system with $D / d=2.2$, was indeed observed experimentally. The results which show density fluctuations at low $Q$ and hence large length scales are consistent with the tendency of more concentrated mixtures to phase separate, and this tendency is enhanced for higher size ratios.

\section{ACKNOWLEDGMENTS}

G. Cinacchi was supported by the EU through a Marie Curie Research Fellowship PIEF-GA-2008-220557 and now by the Ministry of Research of Spain through the Ramón y Cajal contract (Contract. No. RYC-2010-07475). N. Doshi was jointly supported by Imerys and EPSRC DTA. Experiments at ILL were supported by beamtime allocations 9-12216 and 9-10-1044. Materials were kindly donated by AZ Electronics (Klebosol) and Lubrizol (Solsperse 41000). We thank an anonymous referee for suggesting the comparison with Kirkwood-Buff theory of mixtures.

\section{APPENDIX: COMPARISON BETWEEN PRESENT THEORY AND KIRKWOOD-BUFF THEORY OF MIXTURES}

In this appendix, we compare the theory described and validated in the main text with Kirkwood-Buff (KB) theory of mixtures. $^{29,30}$ This comparison is made in terms of the value that the structure factor reaches in the limit of vanishing wave vector. In practice, we will be comparing $\Delta S_{\circ \circ}^{P \circ}(0)$ obtained from Eq. (14) of the main text with the value of this quantity that can be derived by applying the KB theory.

From Eq. (14), we have

$\Delta S_{\circ \circ}^{P \circ}(0)=S_{\circ \circ}^{P}(0)-S_{\circ \circ}^{\circ}(0)=4 \pi \rho_{\circ} \int_{D+d}^{d} d r r^{2} g_{\circ \circ}^{\circ}(r) \Delta v_{I I}(r)$.

In terms of $\mathrm{KB}$ integrals, $G_{\circ \circ}^{\circ}$ and $G_{\circ \circ}^{P}$, defined as

$$
\begin{aligned}
& G_{\circ \circ}^{\circ}=4 \pi \int_{0}^{\infty} d r r^{2}\left[g_{\circ \circ}^{\circ}(r)-1\right], \\
& G_{\circ \circ}^{P}=4 \pi \int_{0}^{\infty} d r r^{2}\left[g_{\circ \circ}^{P}(r)-1\right],
\end{aligned}
$$

the quantity $\Delta S_{\circ \circ}^{P \circ}(0)$ reads

$$
\Delta S_{\circ \circ}^{P \circ}(0)=\rho_{\circ} \Delta G_{\circ \circ}^{P \circ}
$$

with $\Delta G_{\circ \circ}^{P \circ}=G_{\circ \circ}^{P}-G_{\circ \circ}^{\circ}$.

$\mathrm{KB}$ theory relates $\mathrm{KB}$ integrals to the matrix $\mathbf{B}$ of the derivatives of the densities with respect to the chemical potentials. In a general mixture having components $i$ and $j$, we have

$$
B_{i j}=\left(\frac{\partial \rho_{i}}{\partial \beta \mu_{j}}\right)_{T, \mu_{i}}=\rho_{i} \rho_{j} G_{i j}+\rho_{i} \delta_{i j} .
$$

The matrix $\mathbf{B}$ coincides with the inverse of the matrix $\mathbf{A}$ of the derivatives of the chemical potentials with respect to the densities

$$
A_{i j}=\left(\frac{\partial \beta \mu_{i}}{\partial \rho_{j}}\right)_{T, \rho_{i}}
$$

In practice, one has to build the matrix $\mathbf{A}$, invert it to get the matrix $\mathbf{B}$, and then obtain the various $\mathrm{KB}$ integrals.

In order to build the matrix $\mathbf{A}$, one has to have a free energy expression for the mixture. This is the key (and possibly weak) point in the application of the KB theory. In our specific case, we therefore need a free energy expression for a dilute and isotropic mixture of hard spheres and hard discs. As in the main text, we will model the latter as infinitely thin hard platelets. 
Since we are dealing with dilute mixtures, it is reasonable to start assuming a virial expansion of the excess free energy, $F^{e x}$, truncated at the leading order

$$
\frac{\beta F^{e x}}{V}=\beta \Phi^{e x}=\frac{1}{2}\left[\rho_{\circ}^{2} v_{\circ}+2 \rho_{\circ} \rho_{P} v_{\circ P}+\rho_{P}^{2} v_{P P}\right] .
$$

In the above expression, $\Phi^{e x}$ is the excess free energy density, while the quantities $v_{\circ \circ}, v_{\circ}$, and $v_{P P}$ are, respectively, the sphere-sphere, sphere-platelet, and isotropically averaged platelet-platelet excluded volumes.

Equation (A7) can systematically be improved, as $\rho_{\circ}$ and/or $\rho_{P}$ increases, by adding further virial terms. One may shortcut this procedure by adopting an approximate theory that would include effects of the higher order virial terms. One possibility is to modify Eq. (A7) as follows:

$$
\beta \Phi^{e x}=\Psi\left(\rho_{\circ}\right)\left[\rho_{\circ}^{2} v_{\circ}+2 \rho_{\circ} \rho_{P} v_{\circ P}+\rho_{P}^{2} v_{P P}\right] .
$$

The pre-factor $\Psi$, solely depending on the hard sphere density because of the vanishing volume of the hard platelets, derives from the very accurate Carnahan-Starling equation of state for hard spheres ${ }^{32}$ and is equal to

$$
\Psi\left(\rho_{\circ}\right)=\frac{1}{8} \frac{4-3 \rho_{\circ} v_{\circ}}{\left(1-\rho_{\circ} v_{\circ}\right)^{2}},
$$

where $v_{\circ}$ is the volume of one sphere.

One can note that Eq. (A8) is a generalisation to mixtures of Parsons theory. ${ }^{33}$ For monodisperse hard rods, this theory proved successful in accounting for the isotropic-to-nematic phase transition ${ }^{34-37}$ and it was then extended to study mixtures of such particles, ${ }^{38}$ also including smectic phases. ${ }^{39-41}$ For hard discs, the Parsons theory has also been applied, ${ }^{42,43}$ but, as it reduces to Onsager theory ${ }^{44}$ for thin particles, it does not describe the isotropic-nematic phase transition too well in the thin platelet limit.

Other possible choices in place of Eq. (A8) could have been scaled particle theory, ${ }^{45,46}$ or fundamental measure theory. ${ }^{47,48}$ These theories provide a good account of the hard sphere system, ${ }^{45,47}$ while their predictions for the isotropic equation of state of infinitely thin hard platelets were compared to computer simulation data in Refs. 49 and 50.

Since the regime considered in the experiments corresponds to mixtures that are very dilute in platelets but moderately dilute in spheres, we chose to employ Eq. (A8) as it leads to a more accurate description of the pure hard sphere equation of state. It has to be recalled, nonetheless, that its validity, as well as that of the other above-mentioned theories for mixtures, is still to be checked fully.

Once the ideal contribution has been added to an excess free energy expression, chemical potentials as a function of densities can be obtained and from these their derivatives. The matrix $\mathbf{A}$ is then built and its inversion leads to the matrix B, of which we pick the "оo" element, both when platelets are present, $B_{\circ \circ}^{P}$, and when they are not, $B_{\circ \circ}^{\circ}$. From these two terms, the corresponding $\mathrm{KB}$ integrals are obtained according to Eq. (A5) and finally we arrive at $\Delta S_{\circ \circ}^{P \circ}(0)$ via Eq. (A4).

Figure 5 shows a comparison between the two ways of calculating the increase in the value of the structure factor at $Q=0$ when platelets are added. We considered spheres of diameter $d$ and platelets of diameter $D$, respectively, equal to
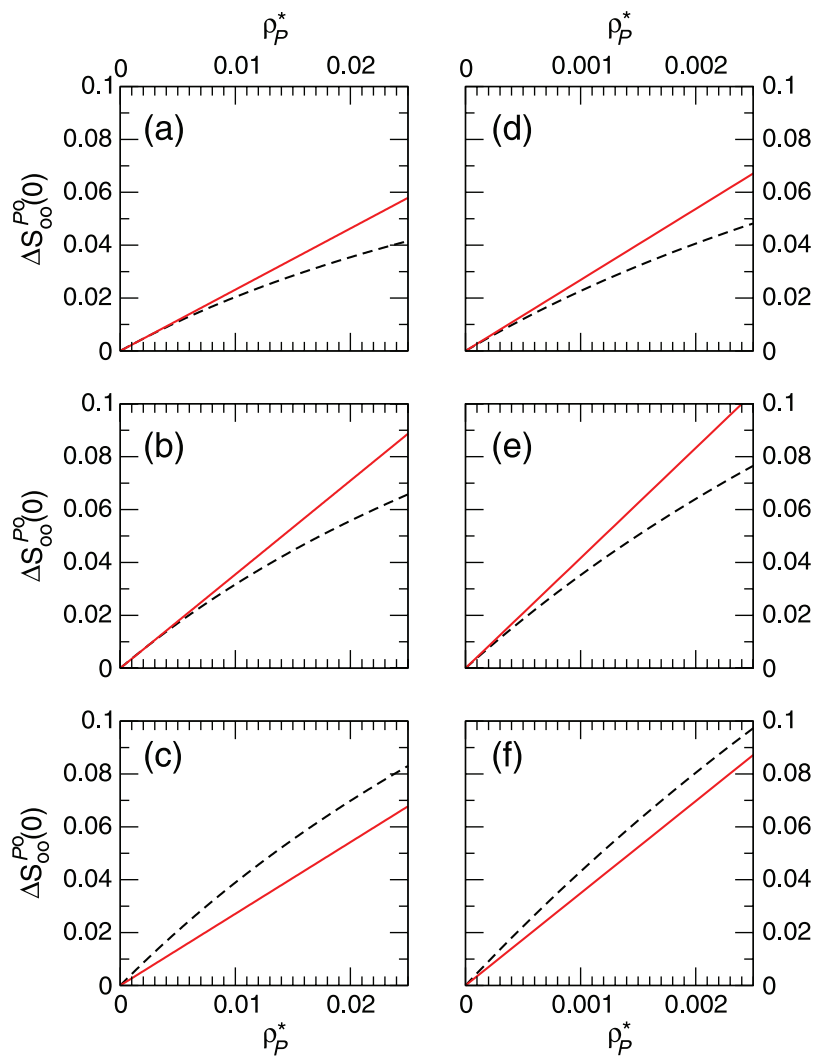

FIG. 5. Comparison between the curves obtained making use of Eq. (1) (solid line) and those coming from KB theory and Eq. (A8) (dashed line): small plate/sphere diameter ratio, $D / d=2 \sqrt{5 / \pi}$, and (a) $\rho_{\circ}^{*}=0.05$, (b) $\rho_{0}^{*}=0.1$, and (c) $\rho_{0}^{*}=0.2$; large plate/sphere diameter ratio, $D / d=4 \sqrt{5 / \pi}$, and (d) $\rho_{\circ}^{*}=0.05$, (e) $\rho_{\circ}^{*}=0.1$, and (f) $\rho_{\circ}^{*}=0.2$.

$2 \sqrt{5 / \pi} d$ and $4 \sqrt{5 / \pi} d$, at three representative values of the reduced density of spheres, $\rho_{\circ}^{*}=\rho_{\circ} d^{3}$, and various values of the reduced density of platelets, $\rho_{P}^{*}=\rho_{P} d^{3}$. The values of $\rho_{\circ}^{*}$ considered were $0.05,0.1$, and 0.2 , the latter corresponding to the actual sphere density in the experiments, while the maximal value of $\rho_{P}^{*}=\rho_{P} d^{3}$ considered depended on the platelet diameter and were in accordance with the maximal platelet density in the experiments. In general, the two corresponding curves agree at low sphere densities, with the slope of the linear curve coming out from current theory coinciding with the limit for $\rho_{P} \rightarrow 0$ of $\Delta S_{\circ \circ}^{P \circ}(0)$ calculated making use of the KB theory plus Eq. (A8). The agreement between the two curves extends up to $\rho_{P}^{*} \sim 0.01$ for smaller platelets, while the two curves depart from one another at much smaller values of $\rho_{P}$ if the platelets are larger. For $\rho_{\circ}^{*}=0.2$, the two curves differ already at low values of $\rho_{P}$, yet they do not depart that much as $\rho_{P}$ increases.

The good agreement between the two curves at low sphere density [Figs. 5(a), 5(b), 5(d), and 5(e)] traces back to the dilution of the mixtures, the most suitable conditions for the validity of both the theory described in the main text and the free energy expression of Eq. (A8). The non-linearity of the curve obtained from KB theory plus Eq. (A8) along with the splitting of the curves corresponding to the two methods on increasing the platelet density [Figs. 5(a), 5(b), 5(d), and 5(e)] are due to platelet-platelet interactions; indeed, this 
latter splitting is more marked for larger platelets, reflecting the increased importance of platelet-platelet interactions as their diameter increases with respect to that of the spheres, at the same values of the density of the two components. As soon as sphere density increases, terms that are proportional to larger powers of the $\rho_{\circ}$ become important and they should be responsible for the differences between the two routes in panels (c) and (f) of Fig. 5, even at low platelet densities.

Experiments suggest that up to the range of sphere and platelet density investigated a linear regime of the changes of the sphere structure factor with density of the platelets holds, in line with the theory presented in the main text. While certain deviations are seen between the two methods at the concentrations relevant to the experiments here, it is gratifying to see that the differences are small, and both approaches agree in the low concentration limit, as expected.

It would be of interest to see how the two routes to get the upswing of the sphere structure factor at $Q=0$ compare with one another and to computer simulations when more concentrated suspensions of hard spheres and hard platelets are considered. Current theory can systematically be extended including platelet-platelet interactions as well as terms corresponding to larger powers of the sphere density. The former would result in the structure factor change no longer being linear in $\rho_{P}$, while the latter terms will be needed as the sphere density increases. This can come at the expense of considering and evaluating, with the required accuracy, progressively more complicated integrals. This task may ultimately end up to a test of the Parsons theory of Eq. (8) and, substituting platelets with rods, of the Parsons theory of sphere-rod mixtures, as well as of the other theories put forward for mixtures of spheres with non-spherical particles. We hope to have a chance to address these points in the future.

${ }^{1}$ N. Doshi, G. Cinacchi, J. S. van Duijneveldt, T. Cosgrove, S. W. Prescott, I. Grillo, J. Phipps, and D. I. Gittins, J. Phys. Condens. Matter 23, 194109 (2011).

${ }^{2}$ J. S. van Duijneveldt, A. W. Heinen, and H. N. W. Lekkerkerker, Europhys. Lett. 21, 369 (1993).

${ }^{3}$ S. M. Ilett, A. Orrock, W. C. K. Poon, and P. N. Pusey, Phys. Rev. E 51, 1344 (1995)

${ }^{4}$ A. Imhof and J. K. G. Dhont, Phys. Rev. Lett. 75, 1662 (1995).

${ }^{5}$ F. M. van der Kooij, M. Vogel, and H. N. W. Lekkerkerker, Phys. Rev. E 62, 5397 (2000).

${ }^{6}$ S. M. Oversteegen, C. Vonk, J. Wijnhoven, and H. N. W. Lekkerkerker, Phys. Rev. E 71, 041406 (2005).

${ }^{7}$ F. Cousin, V. Cabuil, I. Grillo, and P. Levitz, Langmuir 24, 11422 (2008).

${ }^{8}$ N. Yasarawan and J. S. van Duijneveldt, Soft Matter 6, 353 (2009).

${ }^{9}$ D. Kleshchanok, A. V. Petukhov, P. Holmqvist, D. V. Byelov, and H. N. W. Lekkerkerker, Langmuir 26, 13614 (2010).
${ }^{10}$ D. Kleshchanok, J. M. Meijer, A. V. Petukhov, G. Portale, and H. N. W. Lekkerkerker, Soft Matter 7, 2832 (2011).

${ }^{11} \mathrm{~S}$. Asakura and F. Oosawa, J. Chem. Phys. 22, 1255 (1954).

${ }^{12}$ S. Asakura and F. Oosawa, J. Polym. Sci. 33, 183 (1958).

${ }^{13}$ T. G. Mason, Phys. Rev. E 66, 060402 (2002).

${ }^{14}$ D. Kleshchanok, R. Tuinier, and P. R. Lang, J. Phys. Condens. Matter 20, 073101 (2008).

${ }^{15}$ A. Vrij, J. W. Jansen, J. K. G. Dhont, C. Pathmamanoharan, M. M. KopsWerkhoven, and H. M. Fijnaut, Faraday Discuss. Chem. Soc. 76, 19 (1983).

${ }^{16}$ A. P. Gast, C. K. Hall, and W. B. Russel, J. Colloid Interface Sci. 96, 251 (1983).

${ }^{17}$ H. N. W. Lekkerkerker, W. C. K. Poon, P. N. Pusey, A. Stroobants, and P. B. Warren, Europhys. Lett. 20, 559 (1992).

${ }^{18}$ H. De Hek and A. Vrij, J. Colloid Interface Sci. 70, 592 (1979).

${ }^{19}$ H. De Hek and A. Vrij, J. Colloid Interface Sci. 84, 409 (1981).

${ }^{20}$ W. C. K. Poon, F. Renth, R. M. L. Evans, D. J. Fairhurst, M. E. Cates, and P. N. Pusey, Phys. Rev. Lett. 83, 1239 (1999).

${ }^{21}$ C. F. Vester, Colloid Polym. Sci. 84, 63 (1938).

${ }^{22}$ A. Vrij, Pure Appl. Chem. 48, 471 (1976).

${ }^{23}$ K. Mutch, J. S. van Duijneveldt, and J. Eastoe, Soft Matter 3, 155 (2007).

${ }^{24}$ K. Mutch, J. S. van Duijneveldt, J. Eastoe, I. Grillo, and R. Heenan, Langmuir 24, 3053 (2008).

${ }^{25}$ K. Mutch, J. S. van Duijneveldt, J. Eastoe, I. Grillo, and R. Heenan, Langmuir 25, 3944 (2009)

${ }^{26}$ K. Mutch, J. S. van Duijneveldt, J. Eastoe, I. Grillo, and R. Heenan, Langmuir 26, 1630 (2010).

${ }^{27}$ J. A. Barker and D. Henderson, Rev. Mod. Phys. 48, 587 (1976).

${ }^{28}$ E. Meeron and A. J. F. Siegert, J. Chem. Phys. 48, 3139 (1968).

${ }^{29}$ J. G. Kirkwood and F. P. Buff, J. Chem. Phys. 19, 774 (1951).

${ }^{30}$ A. Ben-Naim, Molecular Theory of Solutions (Oxford University Press, Oxford, 2006), Chap. 4.

${ }^{31}$ N. Doshi, "Colloidal mixtures of spheres and plates," Ph.D. dissertation (University of Bristol, Bristol, 2011).

${ }^{32}$ N. F. Carnahan and K. E. Starling, J. Chem. Phys. 51, 635 (1969).

${ }^{33}$ J. D. Parsons, Phys. Rev. A 19, 1225 (1979).

${ }^{34}$ S. D. Lee, J. Chem. Phys. 87, 4972 (1987).

${ }^{35}$ S. D. Lee, J. Chem. Phys. 89, 7036 (1989).

${ }^{36}$ S. C. McGrother, D. C. Williamson, and G. Jackson, J. Chem. Phys. 104, 6755 (1996).

${ }^{37}$ P. J. Camp, C. P. Mason, M. P. Allen, A. A. Khare, and D. A. Kofke, J. Chem. Phys. 105, 2837 (1996).

${ }^{38}$ H. H. Wensink, G. J. Vroege, J. Phys. Condens. Matter 16, S2015 (2004).

${ }^{39}$ G. Cinacchi, E. Velasco, and L. Mederos, J. Phys. Condens. Matter 16, S2003 (2004).

${ }^{40}$ G. Cinacchi, L. Mederos, and E. Velasco, J. Chem. Phys. 121, 3854 (2004).

${ }^{41}$ G. Cinacchi, Y. Martinez-Raton, L. Mederos, and E. Velasco, J. Chem. Phys. 124, 234904 (2006).

${ }^{42}$ H. H. Wensink, G. J. Vroege, and H. N. W. Lekkerkerker, J. Phys. Chem. B 105, 10610 (2001)

${ }^{43}$ H. H. Wensink and H. N. W. Lekkerkerker, Mol. Phys. 107, 2111 (2009).

${ }^{44}$ L. Onsager, Ann. N.Y. Acad. Sci. 51, 627 (1949).

${ }^{45}$ H. Reiss, H. L. Frisch, and J. L. Lebowitz, J. Chem. Phys. 31, 369 (1959).

${ }^{46}$ K. L. Savithramma and N. V. Madhusudana, Mol. Cryst. Liq. Cryst. 74, 243 (1981).

${ }^{47}$ Y. Rosenfeld, Phys. Rev. Lett. 63, 980 (1989).

${ }^{48}$ A. Esztermann, H. Reich, and M. Schmidt, Phys. Rev. E 73, 011409 (2006).

${ }^{49}$ R. Eppenga and D. Frenkel, Mol. Phys. 52, 1303 (1984).

${ }^{50}$ D. L. Cheung, L. Anton, M. P. Allen, A. J. Masters, J. Phillips, and M. Schmidt, Phys. Rev. E 78, 041201 (2008). 